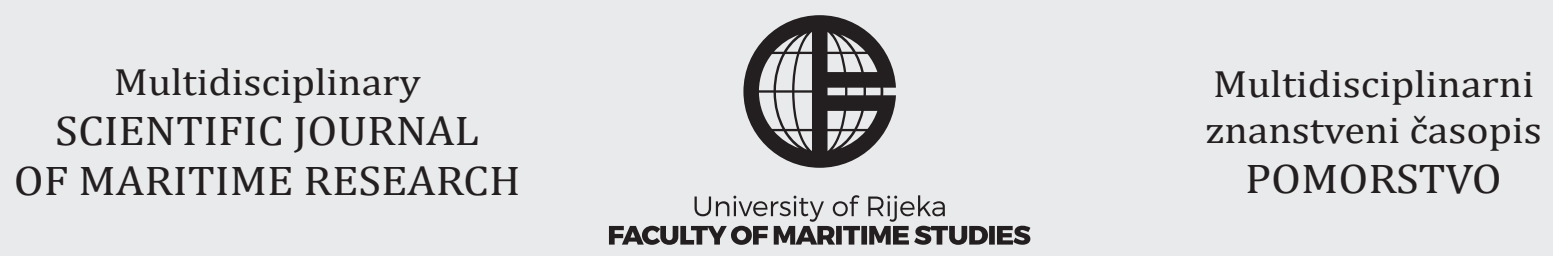

\title{
2D Ship-Like Section Slamming Pressure Numerical Analysis by Conformal Mapping
}

\author{
A. Mertcan Yasa, Abdi Kükner \\ Istanbul Technical University Ayazaga Campus, Faculty of Naval Architecture and Ocean Engineering, 34469, Maslak, Istanbul, Turkey, \\ e-mail: mertcanyasa@gmail.com; kukner@itu.edu.tr
}

\begin{abstract}
In this paper, a method to predict slamming pressures and pressure distribution at the time of water entry for 2D sections is presented. The mathematical model is based on the Schwarz-Christoffel conformal mapping method. This conformal mapping technique has been used to calculate slamming loads during water entry. The pile-up of water during motion is also considered and an alternative pile-up coefficient is assumed against Wagner's generalized method. A simplified and accurate method is presented, which does not include non-linear terms and jet flow in the calculated pressure distribution on monotonically increasing 2D sections like wedge shapes. Comparison with real ship sections has been done to show accuracy of the results. Finally, a simple yet powerful method is obtained to aid the initial design stage of ships.
\end{abstract}

\section{ARTICLE INFO}

Preliminary communication

Received 8 March 2021

Accepted 24 November 2021

\author{
Key words: \\ Wagner \\ Pressure \\ Water entry \\ Slamming \\ Conformal-mapping \\ Initial design
}

\section{Introduction}

One of the biggest challenges of ship design is slamming loads unarguably. Especially the bow flare and bow area of the vessels are under heavy loads and forward slamming becomes a very important parameter to design the structure of the hull such as shell plating, stiffeners and girders. It is a fact that the impact loads are causing not only structural damage and economical loss but also may lead to eventual loss of life at sea. The recent event of M/V NAPOLI incident [5] is a very good example regarding structural failure. Even with the advancing technology, these kinds of incidents show us that simple and direct calculation methods are still in demand. However, this slamming phenomenon has been studied for almost a century. Many methods have been developed to find pressure distribution and slamming loads on ship-like sections as well as more simple shapes such as wedges. At this point, a wedge section is studied to aid ship designers on initial stage to understand the possible slamming loads on the body. The wedge shape is more popular due to its applica- bility to planing hulls, small crafts, trawlers and even freefall lifeboats.

The earliest and most notable studies were done by von Karman [11], who researched the landing of seaplanes, and Wagner [12] who presented an asymptotic solution for small deadrise angles while including water elevation or the so-called pile-up effect. After these initial studies, further research on this specific topic was conducted extensively. Zhao and Faltinsen's 2D boundary element method [14] and the following extension of their studies [15], which included flow separation from knuckles, were very important improvements. Zhao et al. [15] also presented a simplified solution for both wedge shapes and ship-like shapes and compared their results with drop test experiments. Korobkin and Pukhnachov [4] presented a very good study for the initial stage of impact, which can be considered as a review paper of relevant methods and gives a good insight on this specific problem. Mei [7] also developed a similar theory based on conformal mapping techniques and compared his results with Zhao et al. with high accuracy. Mei also developed a new pile-up coef- 
ficient as well as half-breadth estimation with using momentum theorem. It can be stated that his approach took the generalized Wagner method to the next step. In addition to these 2D studies, 3D methods using Navier-Stokes equations were published [9]. Even though the results are in good agreement with experiments, these equations require heavy computational time and resources. There are also ship-like and wedge section experiments such as [13], [1] and [8] and all these studies were developed slamming phenomena greatly.

This study focuses on the development of a very simple yet very powerful method to estimate pressure distribution and pressure loads on 2D sections. Therefore, a new method has been developed with using the conformal mapping technique "Schwarz-Christoffel Method" and a new pile-up coefficient estimated from the initial point of Wagner's study. The results obtained in this study were compared with experiments and Mei's results to confirm accuracy of pressure distribution. For slamming, the comparison has been done against real ship sections and accuracy of results has been discussed. In order to keep this method simple and achieve the aim of this study, non-linear terms and jet flow are neglected.

\section{Theoretical Approach}

In this approach, conformal mapping technique of Schwarz-Christoffel method is used due to it is applicability to fluid dynamics. This method is created for wedgeshaped sections. The wedge section is considered as a diamond on real plane on vertical coordinates as given on left side of the Figure 1 and then mapped into the mapping plane as given on the right side of Figure 1. By forming a diamond shape, the relation between real wedge surface distance $c$ and the mapped distance $c^{\prime}$ is defined. This can be called the first step.

The second step is about calculating the pile-up coefficient. As given on Figure 2, the coordinates were shifted. Now, the bottom half of the diamond is considered as the part that penetrates into the water, and by shifting plates the rise of water surface (or so-called pile-up) can be calculated. The reason of this shift is to calculate pile-up coefficient. The distance on $x$ plane equals to distance on $\eta$ plane, the wedge motion is downward $V$ which is on " $x$ " coordinate and $y$ is the half-breadth of the wedge during the motion; which is denoted as $\xi$ on the mapping plane. On the Figure 1, the conformal mapping is applied to identify

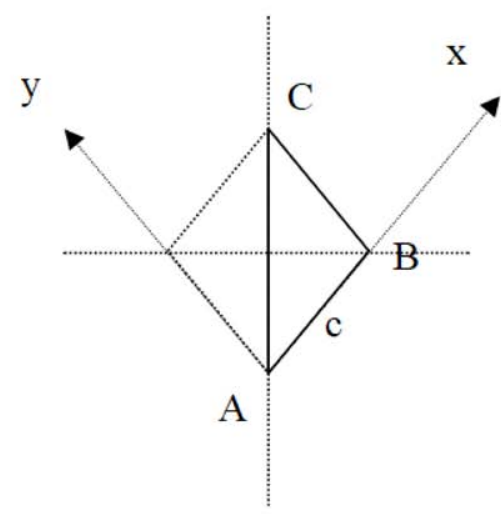

Source: Authors

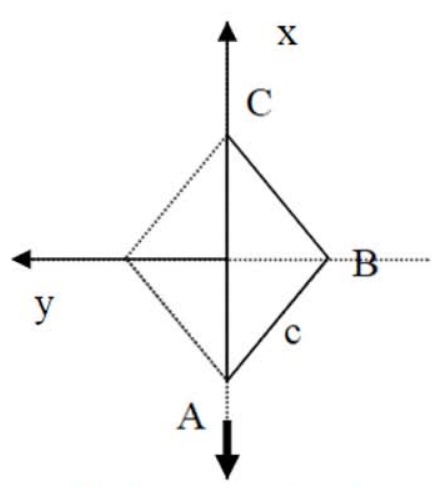

Wedge motion direction

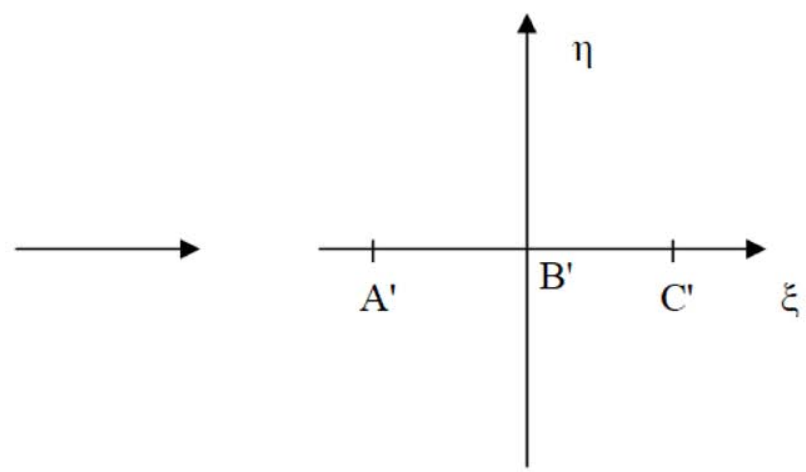

Figure 1 Conformal Mapping Planes at Initial Stage of the Calculations .

Figure 2 Shift of Planes to Calculate Pile-up and Pressure Distribution 
the $c^{\prime}$ value. On the Figure 2, the $\mathrm{x}-\mathrm{y}$ plane is shifted and conformal mapping technique is re-applied to calculate water elevation or so-called pile-up of water.

On the first figure points on wedge $A, B$ and $C$ mapped as $A^{\prime}, B^{\prime}$ and $C^{\prime}$ where distances between $A B$ and $B C$ equal to $c$ and on mapping plane is equal to $c^{\prime}$. $B$ is mapped onto origin point, considered as zero. Then Schwarz-Christoffel general derivative equation is applied.

$$
\begin{aligned}
& f^{\prime}(z)=\frac{d z}{d \zeta}=K\left(\xi-A^{\prime}\right)^{\frac{\alpha}{\pi}-1}\left(\xi-B^{\prime}\right)^{\frac{\beta}{\pi}-1}\left(\xi-C^{\prime}\right)^{\frac{\gamma}{\pi}-1} \\
& F=z=L+K \int\left(\frac{\xi^{2}}{\left(\xi^{2}-c^{\prime 2}\right)}\right)^{1-\frac{\alpha}{\pi}} d \xi
\end{aligned}
$$

On the integral equation, $L$ and $K$ are suitably chosen integral constants [6] and if $z=0$ when $\xi=0$ and this concludes $L=0$ too. When $\xi=\infty, d z=K d \xi$. This makes the constant to be 1 (one). At the end, the generic formula below Eq. 3 is obtained and becomes the foundation of the study.

$f(\xi)=\int\left(\frac{\xi^{2}}{\left(\xi^{2}-c^{\prime 2}\right)}\right)^{1-\frac{\alpha}{\pi}} d \xi$

If the deadrise angle is considered as $\beta$, then the mapping angle can be written as follows.

$\beta=\alpha-\frac{\pi}{2}$

The relation between $c$ and $c^{\prime}$ is found by solving Equation 3 with the usage of hypergeometric series expansion. For all values between zero and $c^{\prime}$, this solution is valid. The results are given on Fig. 3 .

$c=c^{\prime}\left(\frac{\pi}{3 \pi-2 \alpha}\right)$ Hypergeometric $2 \mathrm{~F} 1\left[1-\frac{\alpha}{\pi}, 1.5-\frac{\alpha}{\pi}, 2.5-\frac{\alpha}{\pi} ; 1\right]$
For the calculation of pile-up water, the half-breadth value is necessary. Since the wedge surface on diagonal is denoted as $c$, this makes the half breadth $c^{*} \cos \beta$. Thus, the relation between real plane and mapping plane is used and water elevation (pile-up) is found accordingly. Once the wedge is fully submerged, the maximum half-breadth and maximum water elevation will be achieved. For easy understanding, the maximum pile-up is donated as $C_{P L}$. By using the equations below, the piled-up distance $y$ is calculated.

c. $\cos \beta+y=C_{P L}$

$y=\int_{0}^{\eta}\left(\frac{\eta^{2}}{\eta^{2}+c^{\prime 2}}\right)^{1-\frac{\alpha}{\pi}} d \eta$

And the equation above can be solved as indicated in [2]. It is known that $y$ is the piled-up distance. In order to achieve full breadth (which means half breadth of the submerged wedge plus rise of the water) the equation (9) is written.

$y=c \cdot \int_{0}^{\frac{\eta}{c^{\prime}}}\left(\frac{\left(\frac{\eta}{c^{\prime}}\right)^{2}}{\left(\frac{\eta}{c}\right)^{2}+(B)^{2}}\right)^{1-\frac{\alpha}{\pi}} d \eta / c^{\prime}$

c. $\cos \beta+$ c. $Y=C_{P L}$

$Y$ is the integral part given in Equation (8). The integral is to be solved for each $\eta / c^{\prime}$ value manually. Starting from $\eta / c^{\prime}=0$, the values are defined and integral will be solved and then put into Equation (8) and (9). This solution is continued until obtaining the value of $\pi / 2$, which is the end point of the calculation for zero angle deadrise. Once a specific $\eta / c^{\prime}$ is found to calculate the pile-up coefficient for $\pi / 2$, that specific $\eta / c^{\prime}$ can be applied for all deadrise an-

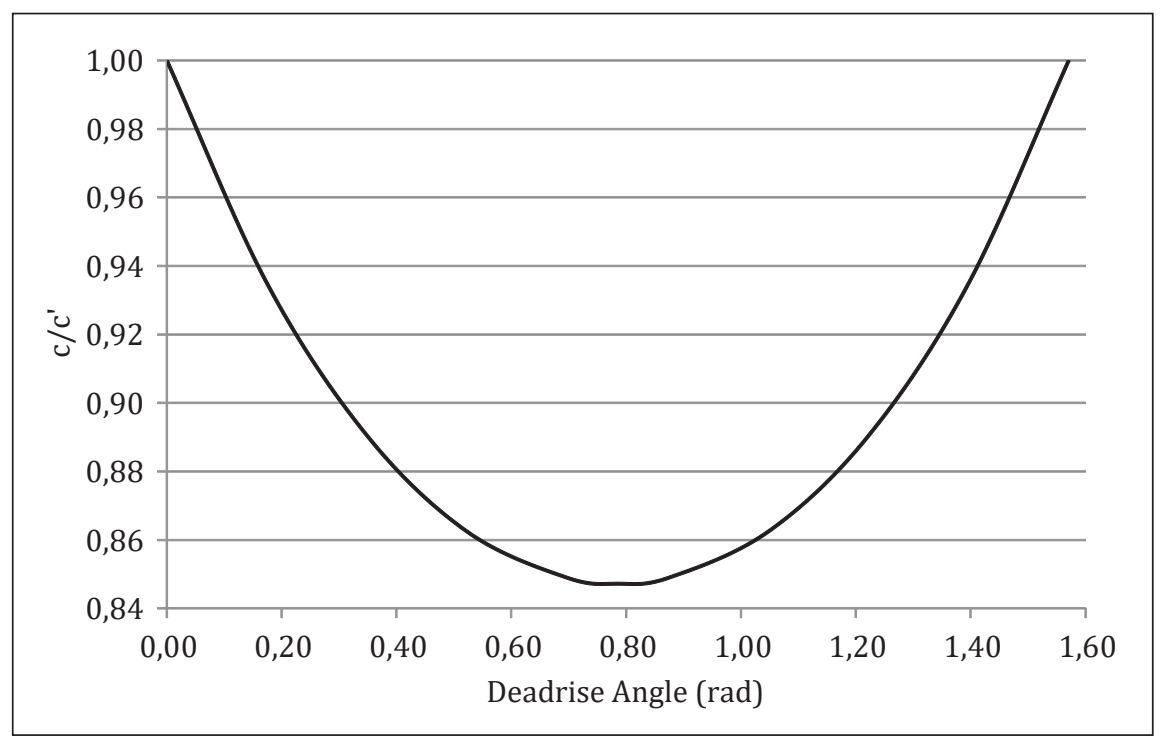

Figure 3 Relation Between c and c' 
Table 1 Pile-Up Coefficient Results

\begin{tabular}{|c|c|c|c|c|c|c|}
\hline DEG $\left.^{\circ}\right)$ & $\mathbf{R A D}$ & $\boldsymbol{\alpha}$ & $\mathbf{c}^{\prime} / \mathbf{c}$ & $\mathbf{y}$ & $\mathbf{\operatorname { c o s } \beta}$ & $\mathbf{\mathbf { C } _ { \mathrm { PL } }} / \mathbf{c}(\boldsymbol{\gamma})$ \\
\hline $\mathbf{0}$ & 0 & 1.570796 & 1 & 0.570796332 & 1.570796 \\
\hline $\mathbf{1 0}$ & 0.17453 & 1.745329 & 1.069649 & 0.588034742 & 0.984808 & 1.572842 \\
\hline $\mathbf{2 0}$ & 0.34907 & 1.919862 & 1.123136 & 0.618190733 & 0.939693 & 1.557883 \\
\hline $\mathbf{3 0}$ & 0.52360 & 2.094395 & 1.159649 & 0.661207814 & 0.866025 & 1.527233 \\
\hline $\mathbf{4 0}$ & 0.69813 & 2.268928 & 1.177995 & 0.71756118 & 0.766044 & 1.483606 \\
\hline $\mathbf{4 5}$ & 0.78540 & 2.356194 & 1.180341 & 0.750865342 & 0.707107 & 1.457972 \\
\hline $\mathbf{5 0}$ & 0.87267 & 2.443461 & 1.17803 & 0.787697087 & 0.642788 & 1.430485 \\
\hline $\mathbf{6 0}$ & 1.04720 & 2.617994 & 1.159555 & 0.872149263 & 0.5 & 1.372149 \\
\hline $\mathbf{7 0}$ & 1.22173 & 2.792527 & 1.123158 & 0.97111551 & 0.34202 & 1.313136 \\
\hline $\mathbf{8 0}$ & 1.39626 & 2.96706 & 1.069633 & 1.08430859 & 0.173648 & 1.257957 \\
\hline $\mathbf{9 0}$ & 1.57080 & 3.141593 & 1 & 1 & & 0 \\
\hline
\end{tabular}

Source: Authors

Table 2 Pile-Up Coefficient Comparison

\begin{tabular}{|c|c|c|c|c|}
\hline Deadrise $\left(\mathbf{D e g}^{\circ}{ }^{\circ}\right)$ & Mei's Study $\boldsymbol{\gamma}$ & Present Study $\boldsymbol{\gamma}$ & von Karman & Wagner \\
\hline $\mathbf{0}$ & 1.5708 & 1.5708 & 1.0000 & 1.5708 \\
\hline $\mathbf{1 0}$ & 1.5555 & 1.5728 & 1.0000 & 1.5708 \\
\hline $\mathbf{3 0}$ & 1.5147 & 1.5272 & 1.0000 & 1.5708 \\
\hline $\mathbf{4 5}$ & 1.4696 & 1.4580 & 1.0000 & 1.5708 \\
\hline $\mathbf{6 0}$ & 1.4017 & 1.3722 & 1.0000 & 1.5708 \\
\hline $\mathbf{8 0}$ & 1.2238 & 1.2580 & 1.0000 & 1.5708 \\
\hline
\end{tabular}

Source: Authors

gles. Considering the applicability of the Wagner's method to flat plates to low deadrise angle plates, this study assumes the maximum point is $\pi / 2$; same as Wagner's pile-up coefficient. Based on this assumption, Table 1 is obtained as the final results. The pile-up coefficient is denoted as $\gamma$.

It is possible to compare these results against Mei's [7] results. It is evident that the results are very close to each other and this indicates that this study approximates an accurate estimation for pile-up coefficient. Table 2 gives this comparison.

\subsection{Pressure Distribution Calculation}

To calculate pressure distribution, boundary conditions are to be defined and velocity potential is to be calculated based on the Bernoulli principle. In this study surface tension, gravity, viscosity, compressibility and air cushion effects are neglected as well as jet flow. It is considered that the liquid inertia dominates these kinds of effects during the water entry. The fluid is assumed to be incompressible, irrotational and inviscid flow. By following the equations below and applying the neglections, the velocity potential found is based on the conformal mapping equations. $\rho\left[\frac{\partial \overline{\mathrm{v}}}{\partial \mathrm{t}}+(\overline{\mathrm{v}} \cdot \bar{\nabla}) \overline{\mathrm{v}}\right]=-\bar{\nabla} \mathrm{p}+\mu \nabla^{2} \overline{\mathrm{v}}-\rho g \overline{\mathrm{k}}$

$\nabla x \vec{v}=0$

$\vec{v}=\nabla \phi$

$\nabla \cdot \vec{v}=\frac{\partial u}{\partial x}+\frac{\partial v}{\partial y}+\frac{\partial w}{\partial z}=0$

$\bar{\nabla} \bar{v}=\bar{\nabla} \phi=\nabla^{2} \phi=\frac{\delta^{2} \phi}{\delta x^{2}}+\frac{\delta^{2} \phi}{\delta y^{2}}+\frac{\delta^{2} \phi}{\delta z^{2}}=0$

$\frac{\delta p}{\delta t}+\vec{\nabla}(\rho \vec{u})=0$

$\frac{p}{\rho}=\frac{\delta \phi}{\delta t}+\frac{1}{2}\left(\bar{\nabla} \phi^{2}\right)$

Velocity potential and pressure are now to be defined. It is possible to find velocity potential in terms of this approach using conformal mapping.

$\phi=V . \xi$

$\frac{\delta \phi}{\delta t}=V \frac{\delta x}{\delta c^{\prime}} \frac{\delta c^{\prime}}{\delta t} \frac{\delta \xi}{\delta x}$ 
By using previously defined equations (1) and (2) and (3), it is possible to calculate velocity potential which is equation (18). Downward velocity is $V(\mathrm{~m} / \mathrm{s}), x(\mathrm{~m})$ is the position on the wedge surface, $t(\mathrm{~s})$ is the time and $\xi$ is the position on the mapping plane. The position $x$ is mapped as $\xi$. If the generic form of Schwarz-Christoffel derivative function is used again, it is possible to find all required terms to find the velocity potential.

$\frac{\delta c^{\prime}}{\delta t}=\frac{\gamma B V}{\sin \beta}$

$\frac{\delta \xi}{\delta x}=\left(\frac{\xi^{2}-c^{\prime 2}}{\xi^{2}}\right)^{1-\frac{\alpha}{\pi}}$

$\frac{\delta x}{\delta c^{\prime}}=\int_{c^{\prime}}^{\xi}\left(\frac{\xi^{2}}{\left(\xi^{2}-c^{\prime 2}\right)}\right)^{1-\frac{\alpha}{\pi}} d \xi$

Using the Leibniz Integration method, equation (21) can be solved easily. It is also known that the relation between $c$ and $c^{\prime}$ is to be used and the ratio $c / c^{\prime}$ is given as $B$ as above. At the end the following equation is calculated. The $F(A)$ part goes to infinity due to Leibniz application and is considered as the asymptotic term.

$$
\begin{aligned}
\frac{\delta \phi}{\delta t}= & V\left((2)\left(1-\frac{\alpha}{\pi}\right) c^{\prime} \int_{c^{\prime}}^{\xi}\left(\frac{\left(\xi^{2}\right)^{1-\frac{\alpha}{\pi}}}{\left(\xi^{2}-c^{\prime 2}\right)^{2-\frac{\alpha}{\pi}}}\right) d \xi-\mathrm{F}(\mathrm{A})\right) . \\
& .\left(\frac{\xi^{2}-c^{\prime 2}}{\xi^{2}}\right)^{1-\frac{\alpha}{\pi}} \cdot \frac{\gamma B V}{\sin \beta}
\end{aligned}
$$

In this equation, $F(A)$ is the asymptotic function found from equation (21). In order to solve this asymptotic function, it is assumed to have the similar integration form used for the pile-up coefficient calculation. In order to achieve this, $\xi / c^{\prime}$ values must be defined in terms of $x / c$ on real plane. So, the water reaches the certain point on wedge surface, which is $x$, and $c$ is the total distance of the wedge surface. At a given time; $x / c$ gives the water elevation position. And $\xi / c^{\prime}$ is this definition on the mapping plane. It is known that when $x / c=0$, the water is at the apex, and when $x / c=1$; is where piled up water touches the wedge side. Following Fig. 4 is the result of relation between $\xi / c^{\prime}$ and $x / c$.

And the asymptotic function can be defined for different deadrise angles to improve the results. For example,

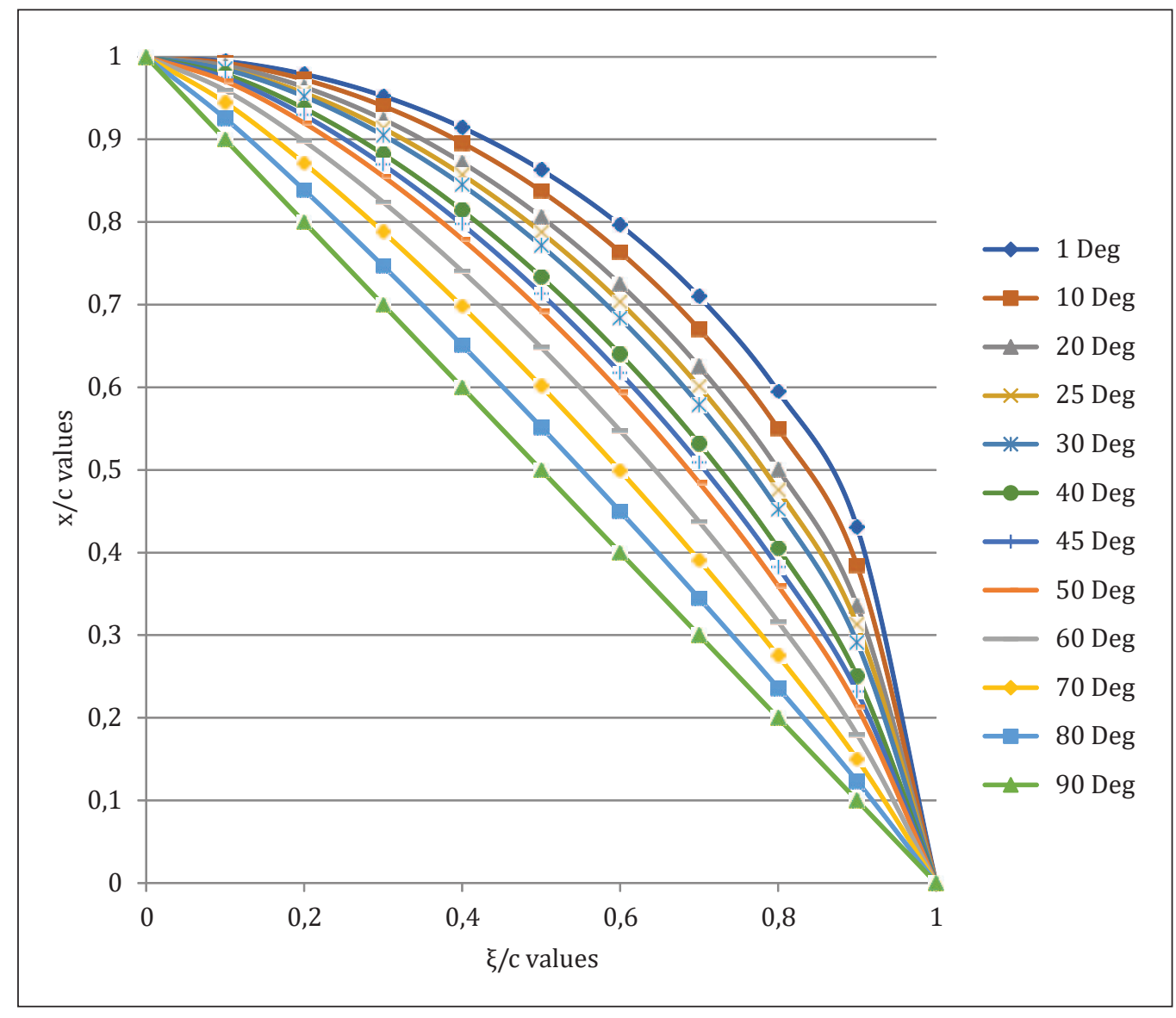

Figure 4 Graphical Representation of the Relation of $\xi / c^{\prime}$ and $x / c$ 


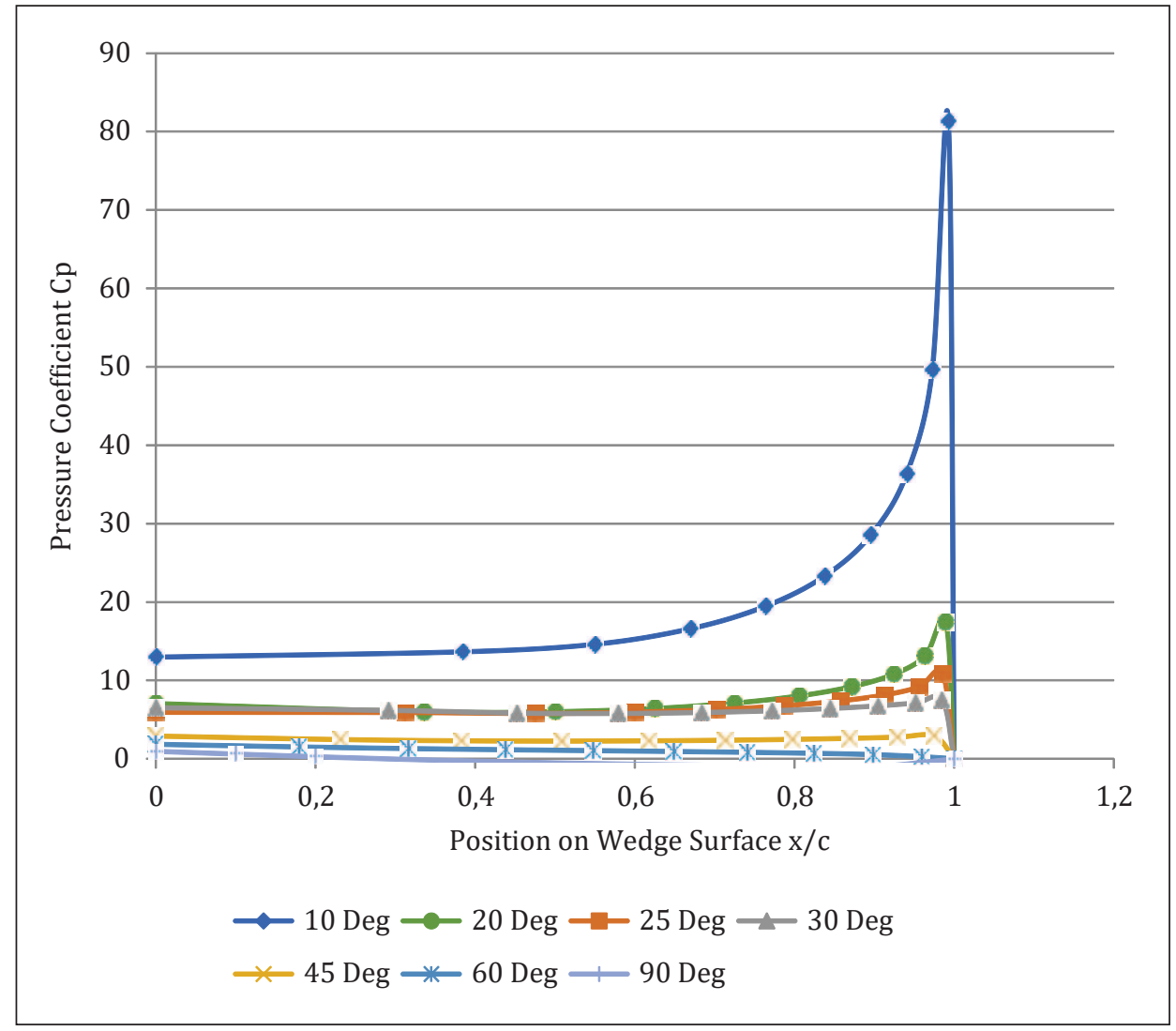

Figure 5 Pressure Coefficient Results for Various Deadrise Angles

Source: Authors

for $1^{\circ}, 10^{\circ}, 20^{\circ}$ degrees of deadrises the asymptotic function is as follows:

$\frac{\left(0.5\left(\frac{\xi}{c^{\prime}}\right)^{2}\right)^{-1-2 \frac{\alpha}{\pi}}}{\left(1^{2}-\left(\frac{\xi}{c^{\prime}}\right)^{2}\right)^{1-\frac{\alpha}{\pi}}}$

Similar functions can be found for all deadrise angles. Once this is completed, the results are obtained as given Fig. 5.

\section{Comparison Against Experiments}

Presented study is validated against Yettou's [13] experiment. Yettou used a wedge with a 25 degrees deadrise angle and carried out drop tests. In this study, the velocity changes for every time step. It is assumed that the wedge is divided into equal sections as given in $x / c$ values. For each corresponding $x_{n}$ and $c_{n}$ values, the wedge penetrates into water. This means that for velocity of $\mathrm{V}_{1}$, there is $\mathrm{x}_{1}$ and $\mathrm{c}_{1}$. For velocity of $V_{n^{\prime}}$ there is $x_{n}$ and $c_{n}$. In this study, $n=11$ and all values are given based on corresponding deadrise angle in Figure 4. The motion of the wedge is essentially calculated in 11 different steps, from touching to the water surface and becoming fully submerged.
For ease of calculation, all of the time stamps and corresponding velocities can be obtained from Yettou [13]. In the Table 3, the time stamps and pressure values are from the experimental readings. The difference on the impact, is that the readings are started on the pressure transducer. This means that the first reading will occur on first transducer, which happens when the wedge is already inside of water. In this current study, the impact pressure at the time of wedge apex touching the water can also be calculated.

It can be observed that at the initial stages, the pressure values are almost identical with each other. This current study approximates the pressures remarkably well at the beginning of the motion. However, when the wedge penetrates through the water or when the time increases, the deviation between experiment and current study gets bigger. There could be two reasons for this error. The first reason being that this study can calculate the pressure values for each velocity: for each $x_{n}(\mathrm{~m})$, there is a corresponding $V_{n}$ (wedge velocity, m/s). However, between each step, velocity is assumed to be constant. Between $\mathrm{x}_{1}$ and $\mathrm{x}_{2}$, the velocity does not change. They are fixed velocities as $V_{1}$ and $V_{2}$. Therefore, the velocity does not fully vary and this may cause a problem. The second reason being that this study neglects non-linear terms, added mass and jet flow. Therefore, especially when the added-mass terms 
Table 3 Comparison Between Present Study and Yettou's Experiment

\begin{tabular}{|c|c|c|c|}
\hline \multicolumn{2}{|c|}{ Yettou's Experiment (Yettou et al., 2006) } & Current Study \\
\hline $\mathbf{t ~ ( s )}$ & \multicolumn{2}{|c|}{ Pressure (psi) } & Pressure (kPA) \\
\hline 0.0000 & \multicolumn{2}{|c|}{ At the moment of impact } & 141.66 \\
\hline 0.0028 & 19.23 & 132.59 & 138.31 \\
\hline 0.0058 & 18.14 & 125.07 & 127.42 \\
\hline 0.0094 & 14.88 & 102.59 & 71.49 \\
\hline 0.0132 & 12.69 & 87.49 & 47.23 \\
\hline 0.0176 & 9.80 & 67.57 & 32.39 \\
\hline 0.0228 & 7.39 & 50.95 & 26.75 \\
\hline 0.0288 & 6.10 & 42.06 & 18.27 \\
\hline 0.0370 & 4.84 & 33.37 & \\
\hline
\end{tabular}

Source: Authors

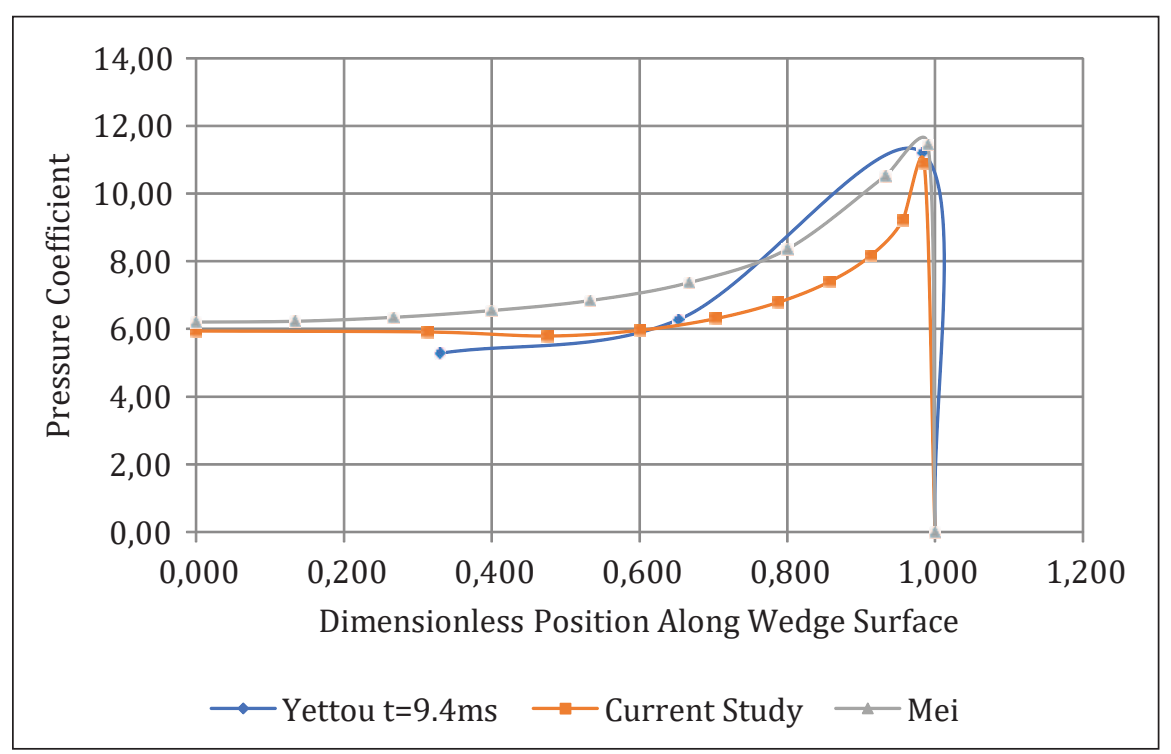

Figure 6 Pressure Coefficient Comparison between Yettou 0.0094s, Presented Study and Mei

Source: Authors

get more dominant, the error gets bigger. This is acknowledged as the shortcoming of this method. However, all things considered, these results are accepted and regarded as successful assumption.

In the same sense, another comparison is shown for the same study. In Yettou's [13] paper, the results were compared against Mei's [7] results. Mei's results are important because his study also uses conformal mapping. As given on Figure 6, the maximum pressure coefficients are almost identical.

\section{Comparison Against Real Ship Sections}

As given by the reference [3], it is possible to define alternative shapes for slamming pressure calculation. The slamming calculation method presented here can only handle certain section shapes (such as wedges). However, the section of a car carrier is nowhere near the shape needed for calculation with this method. Therefore, as per below Fig. 7, alternative shapes are proposed. In the original study [3], there were three alternatives proposed (in the color black) as opposed to the original shape. In this study, there are also three alternatives proposed. The alternative one is marked with the color blue and the dashed line, alternative two is marked with the color red and the dotted line. Alternative three is marked with direct line in green color.

In the study [3], the reference velocity at the time of impact is given as $6.0 \mathrm{~m} / \mathrm{s}$ as wave height is 5 meters. Considering the heading of $0^{\circ}$, the following Fig. 8 is the experimental pressure results for these values. It can be seen that for $5 \mathrm{~m}$ wave height, the density of pressure readings is around 55-60 kPA for lower panel and around 55kPA for upper panel. 


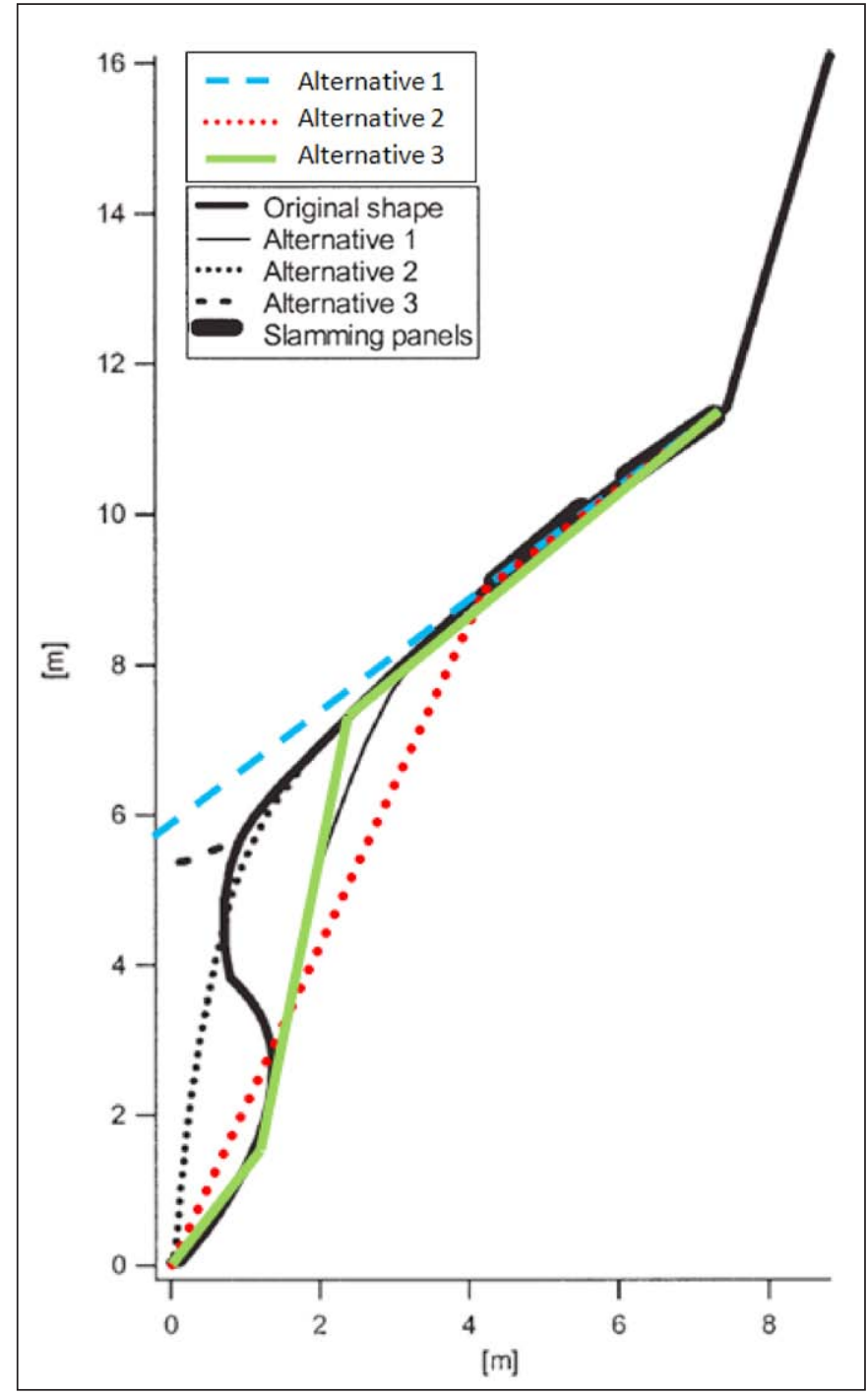

Figure 7 Alternative Shapes
Based on the ship section, it is calculated that the middle point of lower panel is $6.25 \mathrm{~m}$ (over surface, equals to hypotenuse) and upper panel's middle point is $8.50 \mathrm{~m}$ in the same manner. These are the values found via given ship section. For alternative 1, full length of the section surface is $9.53 \mathrm{~m}$ and for alternative 2 ; it is $4.13 \mathrm{~m}$.

It has been considered that both panels are in the water (water rises to the full extent and touches both panels). And following results were obtained as given on Figure 9.

For alternative 1, lower panel gives approximately $63 \mathrm{kPA}$ and upper panel gives approximately 105kPA. Due to the calculation method and alternative form, even though the lower panel gives very good estimation, the upper panel is almost twice the amount of the experimental results.

For alternative 2, lower panel gives approximately $44 \mathrm{kPA}$, and upper panel gives approximately $43 \mathrm{kPA}$. Alternative 2 gives lower estimation, however, and is relatively closer to the actual condition. Considering the shape of the proposed alternatives, alternative 2 gives very realistic results. Secondly, this calculation can be made with an addendum. In Figure 9 above, the lower panel pressure is read when both panels are fully submerged. In order to make a better estimation, the pressure over the surface must be calculated separately for lower panel, when only that panel is fully submerged. The middle point of the panel gives approximately 43kPA pressure which is very close to the above assumption. Figure 10 is the graphical representation of lower panel only result.

Even though the results are lower than the approximate average value from the experiment, they are still within the range of experimental readings as can be seen in Figure 8 above. The readings are between 45 to $75 \mathrm{kPA}$ for lower panel, and 40 to $70 \mathrm{kPA}$ for upper panel (approximate values, read from the graph only). In conclusion, there is a slight underestimation between real values and presented study, but still in the acceptable range. On

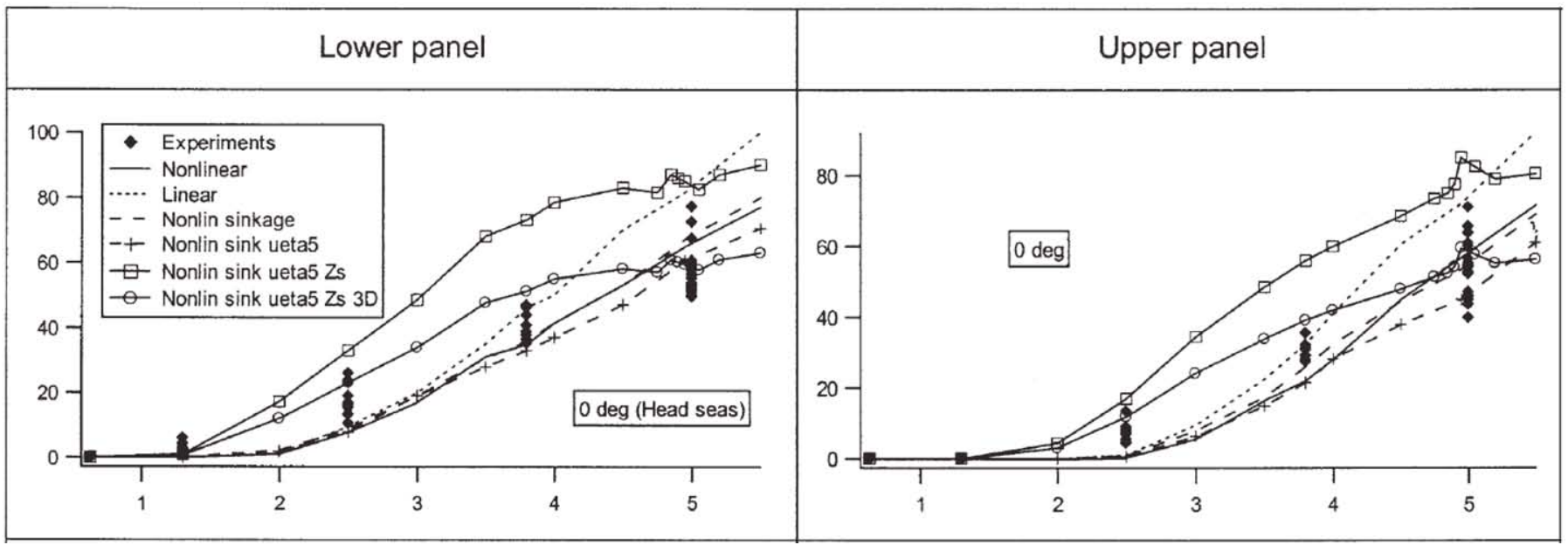

Figure 8 Experimental Results (Black Dots) for Ro-Ro Slamming Experiment 


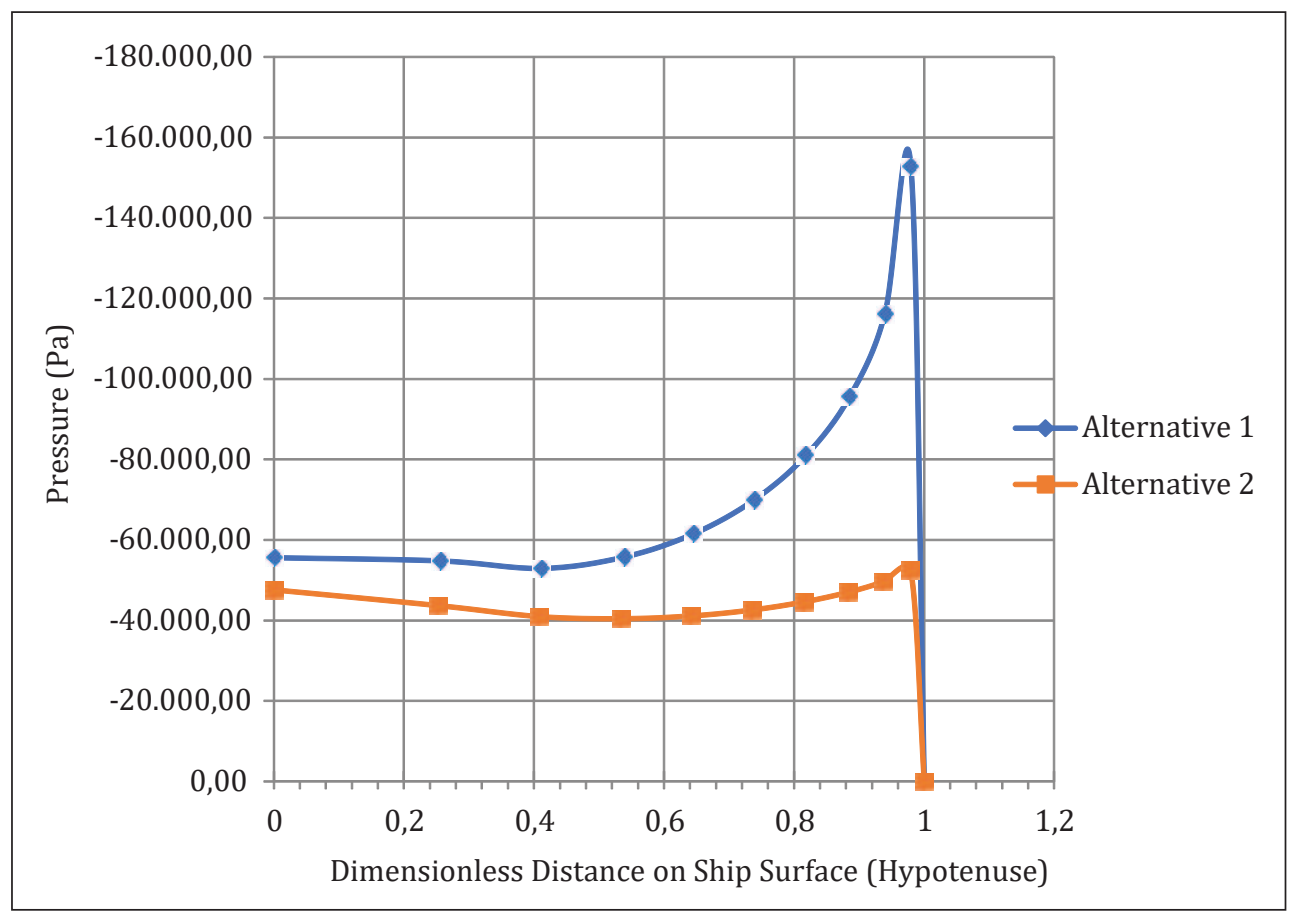

Figure 9 Present Study Results when ship section is fully in water

Source: Authors

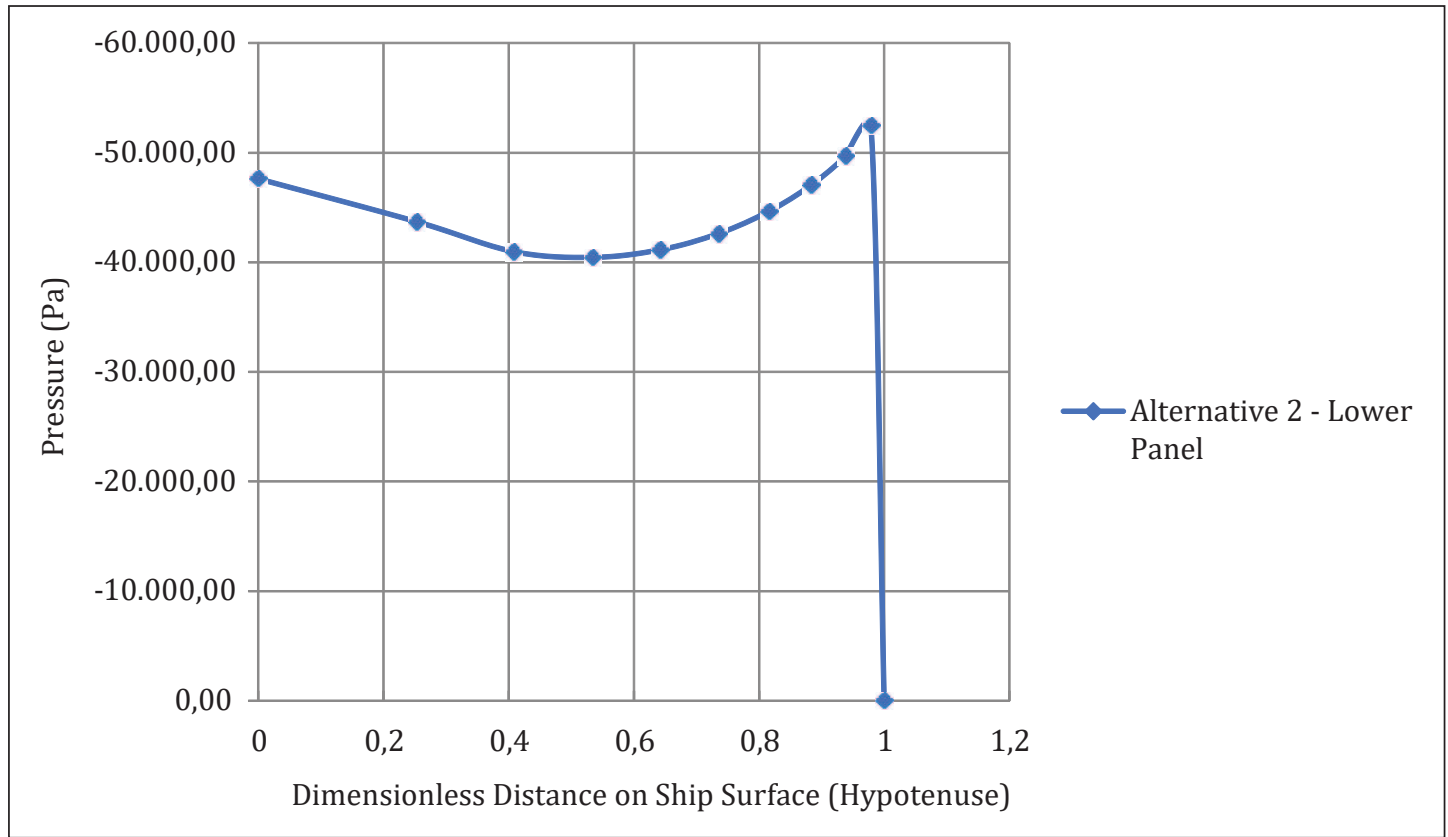

Figure 10 Lower Panel Only

Source: Authors

another note, the current study estimates the maximum pressure at the end of the surface. And if the maximum pressures are considered, for the lower panel, it is $52.6 \mathrm{kPA}$ while for upper panel it is $52.4 \mathrm{kPA}$ for alternative 2 . These results are much closer to the experimental values. Alternative 1 maximum value completely overestimates the upper panel results.
Also, alternative 3 is considered at this point. Alternative 3 is closer in shape to the original, but still ignores the bulbous bow area. Alternative 3 lower panel middle section has around 52kPA and upper panel is around $63 \mathrm{kPA}$. These results are much more realistic and closer to the experimental values given in Fig. 8. Alternative 3 results are given on Figure 11. 


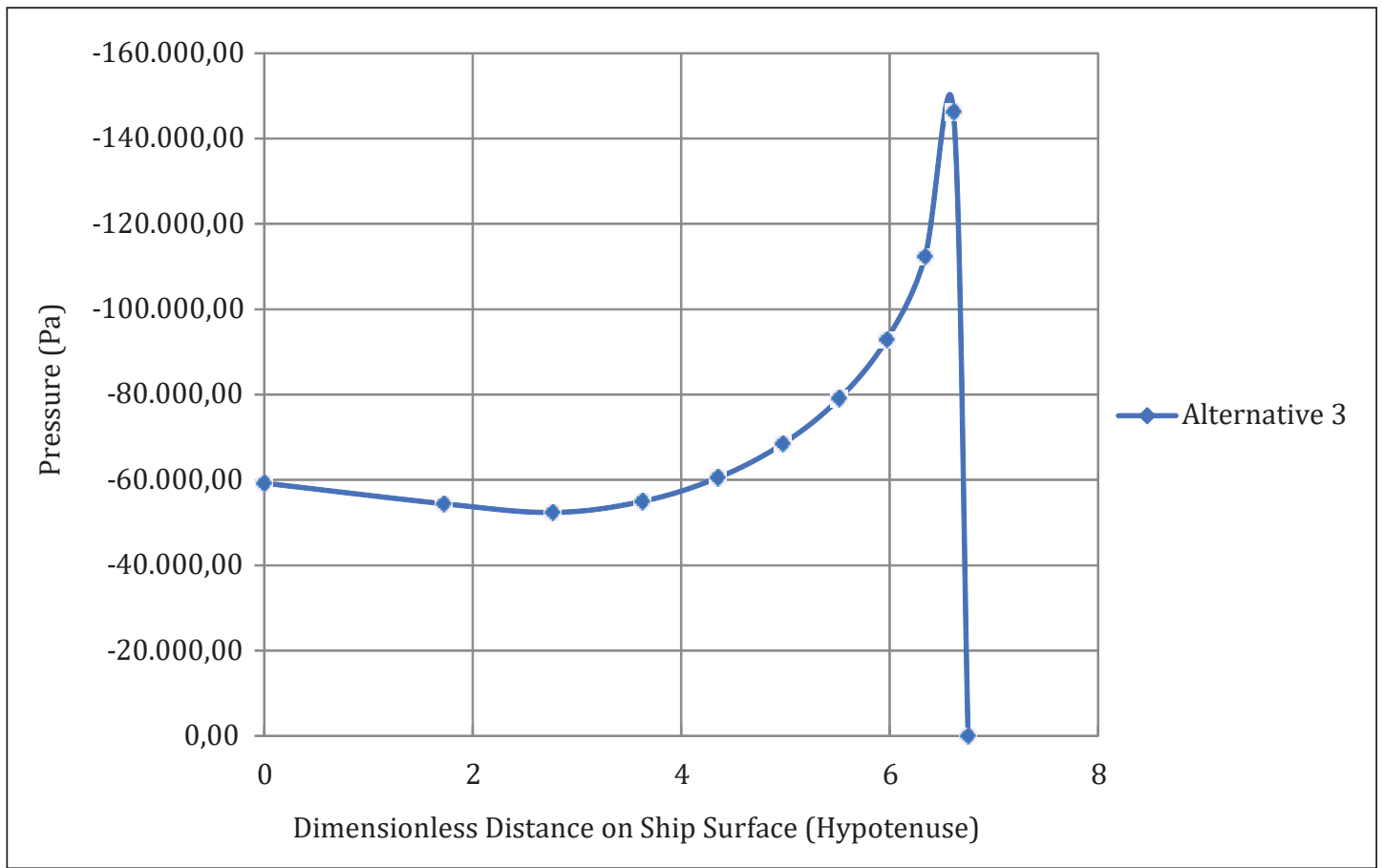

Figure 11 Alternative 3 Results

Source: Authors
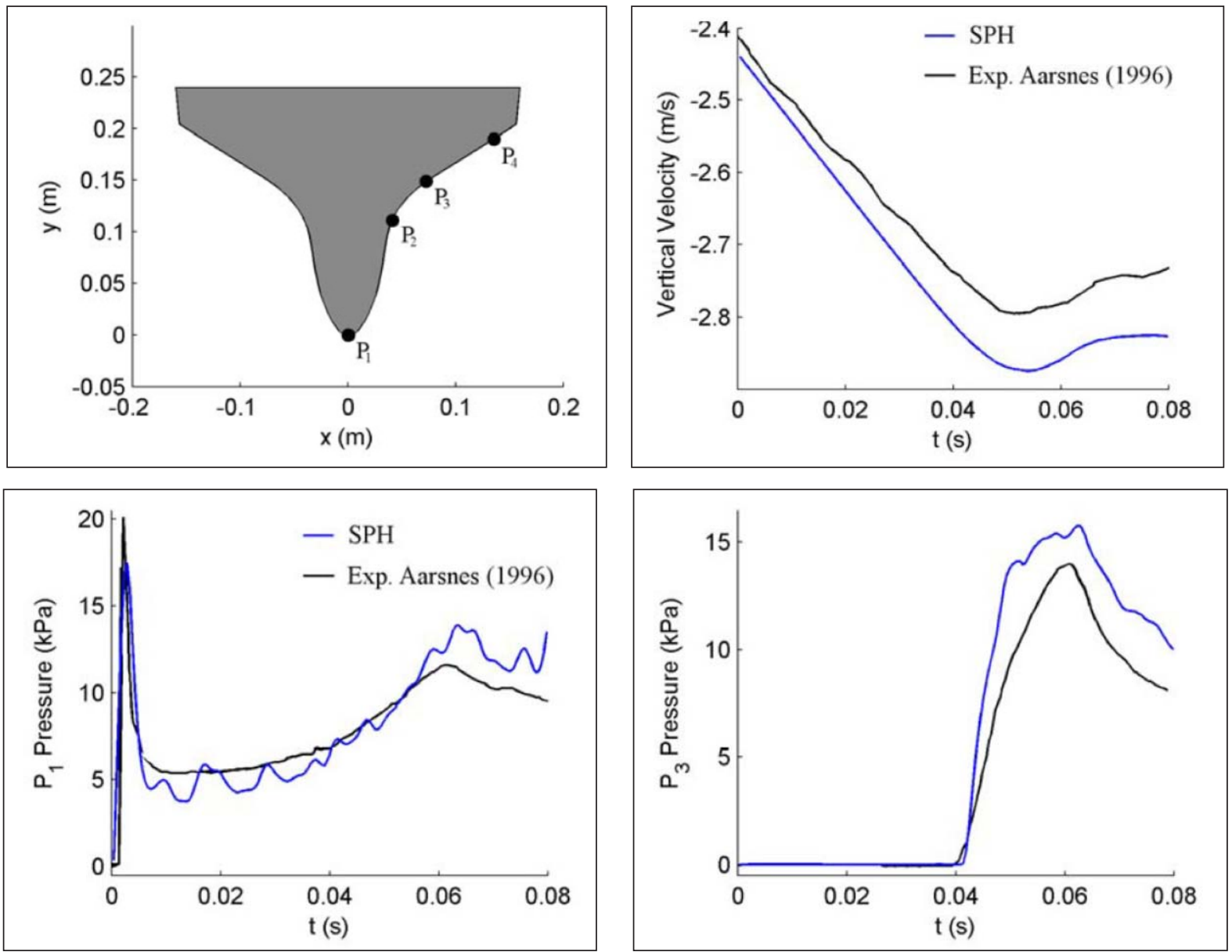

Figure 12 Experiment Results and Section 
However, the maximum point is $146 \mathrm{kPa}$ and this is more than calculated. The reason in this spike is due to calculation method. This presented method in this study handles the section as an individual wedge and pressure is expected to be maximum when water touches the wedge surface. For each individual time step, water touches a certain point on the wedge and that location gives the maxi-

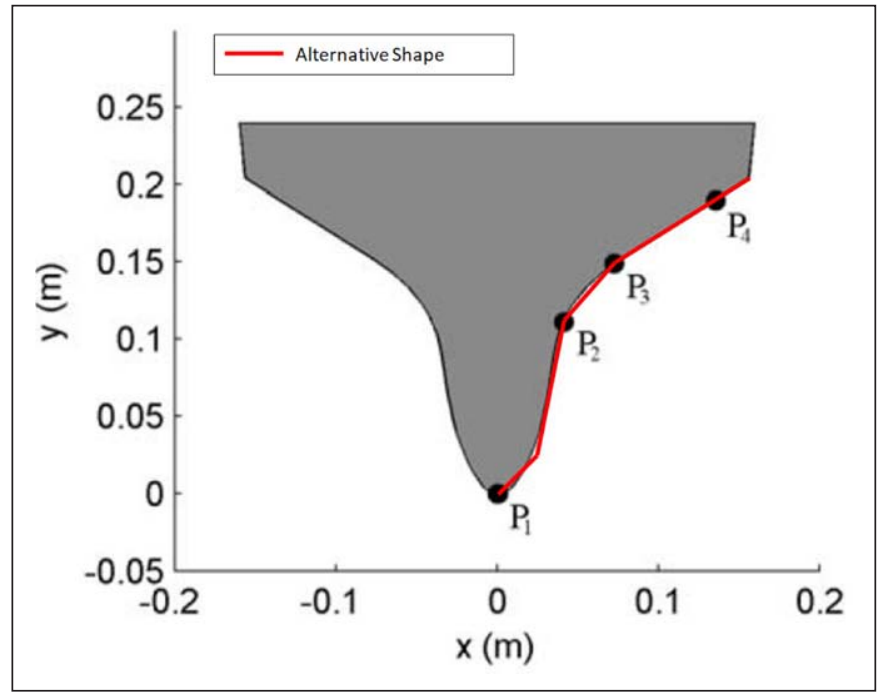

Figure 13 Alternative Shape mum pressure. In this approximation method, the water reaches the end of panel 2, which is the end of that section as given in Fig. 7. This is also same situation for alternative 1 where the maximum pressure is around 152kPA. In any case, alternative 3 gives the best results for readings at panel and more detailed information or further testing is required for further comparison.

Based on the results achieved above, it is determined another comparison must be made to show the applicability of this study. In paper [10], the ship-bow section drop test experiment by Aarsnes (1996) is given. Since we are only interested in experimental values, in the figures given below, SPH values (marked with the color blue) are disregarded and only experiment results are taken into account. Below Figure 12 is the experiment details and Figure 13 is the ship section with proposed alternative section in this study.

In the drop test experiments, the initial velocity is around $2.425 \mathrm{~m} / \mathrm{s}$ (approximately) and maximum pressure at P1 point is $20 \mathrm{kPA}$. Considering the alternative shape proposed in this study, following results were obtained, plotting $21 \mathrm{kPA}$ maximum pressure at $0.0049 \mathrm{~s}$, which is almost identical with the drop test results. For P3, the result of $16.8 \mathrm{kPA}$ at $0.048 \mathrm{~s}$ was estimated against the experimental result of $14.5 \mathrm{kPA}$ at $0.06 \mathrm{~s}$. Considering both P1 and P3 of which are results that can be considered accurate and acceptable.

Source: Authors

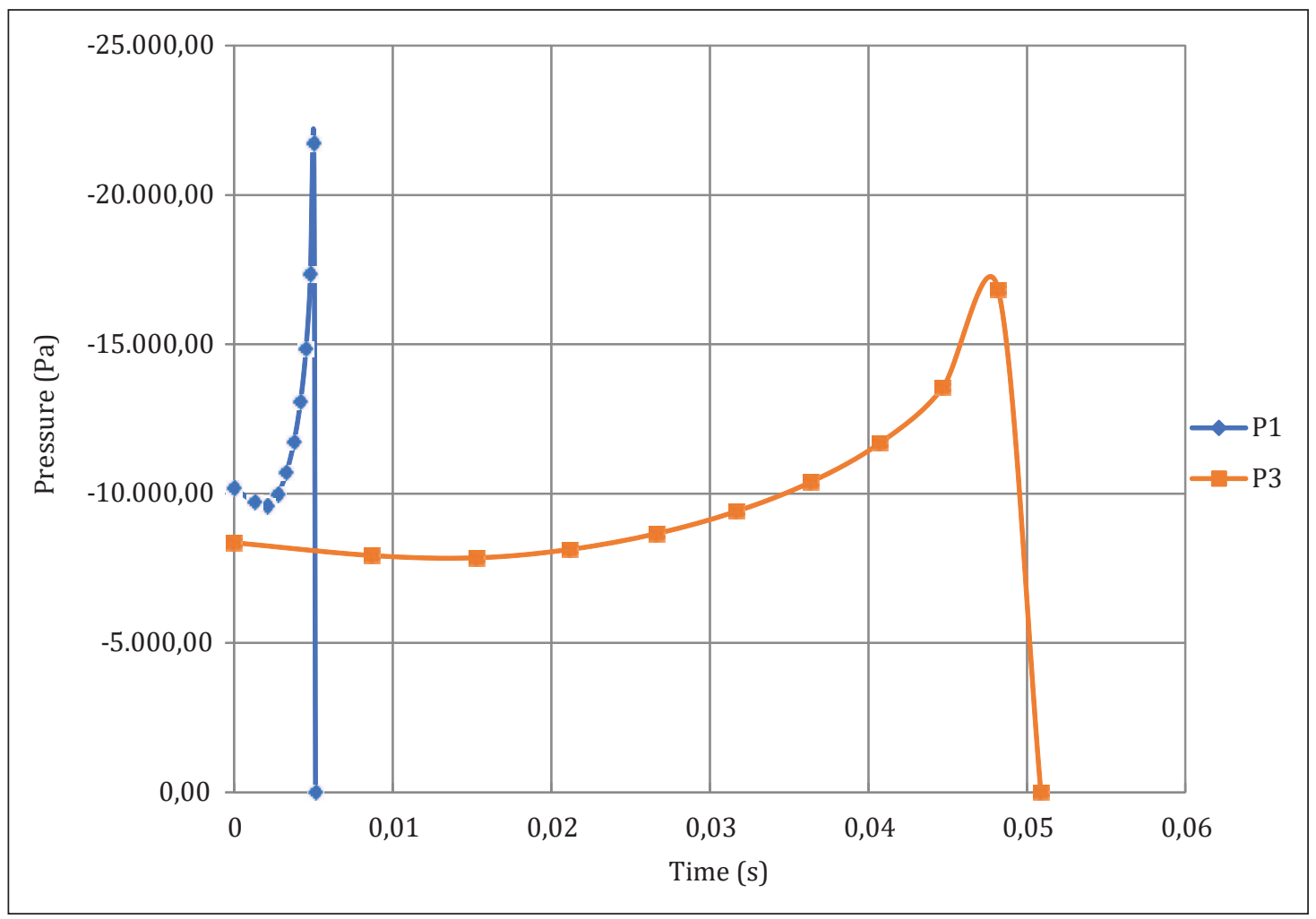

Figure 14 Results from Present Study for Bow Section Pressure Point P1 and P3 
In conclusion, a comparison has been done between present study and real ship sections. First experiment is the real ship values and second experiment is the drop test for ship-like sections. For both cases, alternative sections were proposed and applied accordingly. The results are within acceptable range and very close to the experimental values. Considering that only "similar" sections are taken into account in this study due to its applicability to certain shapes, the results are very encouraging. Therefore, it is concluded that given the correct choice of alternative shape, this study can be applied to real ships too.

\section{Conclusion}

A new method to predict pressure distribution along the surface has been implemented and proposed in this study, as well as the slamming pressures on ship-like sections including a real ship [3] and slamming pressures during drop test experiments [10] compared against the results. The presented method - despite its shortcoming of an applicability to monotonically increasing shapes- is very accurate. The comparison of the results has proven well. This method can not only be used for wedge-like sections but also can be generalized for application to ship section during the initial stage of ship design.

In this study jet flow is not included. Jet flow could be added to improve accuracy of the results. These results are applicable to 2D only. Adding 3D effects as a coefficient or additional term could result in better prediction for bow flare slamming which is considered as a future work. Additionally, the comparison can be done for further alternative sections to improve accuracy and more ship like sections can be considered.

Funding: The research presented in the manuscript did not receive any external funding.

Author Contributions: Research: A. Mertcan Yasa; Verification: Abdi Kükner; Mathematical Harmonization: A. Mertcan Yasa, Abdi Kükner; Writing: A. Mertcan Yasa, Abdi Kükner.

\section{References}

[1] Arai M., Matsunaga K. (1989). Journal of the Society of Naval Architects of Japan, Volume 1989, Issue 166, pp. 343-353.
[2] Ferdinande V. (1966). "Theoretical Consideration on the Penetration of a Wedge into the Water". International Shipping Progress, Vol. 13, No. 140, doi: 10.3233/ISP-19661314002.

[3] Hermundstad A. O., Moan T. (2005). Numerical and experimental analysis of bow flare slamming on a Ro-Ro vessel in regular oblique waves, J Mar Sci Technol 10:105122.

[4] Korobkin A. A., Pukhnachov V.V. (1988). "Initial Stage of Water Impact”, Ann. Rev. Fluid Mech. 20, pp. 159-185, https://doi.org/10.1146/annurev.fl.20.010188.001111.

[5] Marine Accident Investigation Branch (2008). "Report on the Investigation of the Structural Failure of MSC NAPOLI", Report No 9/2008.

[6] Mathews J.H., and Howell R. W. (2012). COMPLEX ANALYSIS: for Mathematics and Engineering, (Sixth Edition), Jones \& Bartlett Learning, Sudbury, MA 01776.

[7] Mei X., Lui Y., Yue D. K. P. (1999). "On the water impact of general two-dimensional sections", Applied Ocean Research 21, pp. 1-15, https://doi.org/10.1016/S0141-1187(98) 00034-0.

[8] Ochi K. (1958). Model experiments on ship strength and slamming in regular waves. Transactions of the Society of Naval Architects and Marine Engineers 66, pp. 345-383.

[9] Sames PC, Schellin TE, Muzaferija S, et al. (1998). Application of a two-fluid finite-volume method to ship slamming. In: Proceedings of the 17th International Conference on Offshore Mechanics and Arctic Engineering (OMAE'98), Lisbon.

[10] Veen D., Gourlay T. (2012). A combined strip theory and Smoothed Particle Hydrodynamics approach for estimating slamming loads on a ship in head seas. Ocean Engineering, Vol. 43, pp. 64-71.

[11] von Karman T. (1929). "The impact of seaplane floats during landing", Technical Report TN-321 (National Advisory Committee for Aeronautics (NACA)).

[12] Wagner, H. (1932). "Über Stross-und Gleitvorgänge an der Oberfläche von Flüssigkeiten”, Z. Angew. Math. Mech. 12, pp. 193-215, https://doi.org/10.1002/zamm.19320120402.

[13] Yettou E.M., Desrochers A., Champoux Y. (2006). "Experimental study on the water impact of a symmetrical wedge", Fluid Dynamics Research 38, pp. 47-66, https://doi. org/10.1016/j.fluiddyn.2005.09.003.

[14] Zhao R. and Faltinsen 0. (1993). "Water entry of twodimensional bodies", J. Fluid Mech., 246, pp. 593-612, http://resolver.tudelft.nl/uuid:fc72f40d-88fa-44eb-a6036ef277ac10d2.

[15] Zhao R., Faltinsen O., Aarsnes J. (1996). "Water Entry of Arbitrary Two-Dimensional Sections with and Without Flow Separation", 21th Symposium of Naval Hydrodynamics Proceedings, Trondheim, Norway, http://resolver.tudelft.nl/ uuid:563b1429-9bab-454b-a029-243abcceee2f. 